Vol. 39 (1989) [361-367]

\title{
DEFORMATIONS OF RINGS
}

JOE YANIK

\begin{abstract}
Let $A$ and $A_{0}$ be rings with a surjective homomorphism $A \rightarrow A_{0}$. Given a flat extension $B_{0}$ of $A_{0}$, deformation of $B_{0} / A_{0}$ over $A$ is a flat extension $B$ of $A$ such that $B \otimes_{A} A_{0}$ is isomorphic to $B_{0}$. We show that such a deformation will exist if $A_{0}$ is an Artin local ring, $A$ is noetherian, and the homological dimension of $B_{0}$ over $A_{0}$ is $\leqslant 2$. We also show that a deformation will exist if the kernel of $A$ is nilpotent and if $A_{0}$ is a finitely generted $A_{0}$-algebra whose defining ideal is a local complete intersection.
\end{abstract}

\section{INTRODUCTION}

The purpose of this paper is to examine some special cases of the following general question. Given rings $A$ and $A_{0}$ with surjection $u: A \rightarrow A_{0}$ and a flat $A_{0}$-algebra $B_{0}$, when does there exist a flat $A$-algebra $B$ such that $B \otimes_{A} A_{0}$ is isomorphic to $B_{0}$ as an $A_{0}$-algebra? If it exists, such a $B$ is called a deformation of $B_{0} / A_{0}$ over $A$. An important case is when $\operatorname{ker}(u)$ is nilpotent. In this case $B$ is said to be an infinitesimal deformation.

One general situation in which a deformation always exists is when $u$ admits a retraction $t: A_{0} \rightarrow A$ (that is $u \circ t=1$ ). In this case $B=B_{0} \otimes A_{0} A$ is a deformation, called the trivial deformation. This is the usual setting in algebraic geometry with $A_{0}$ a field and the problem is to find the structure of the set of nontrivial deformations.

In this paper, however, we take the ring-theoretic approach and consider the general question of when any deformation exists at all. We prove that a deformation exists in two general cases. One is when $A_{0}$ is an Artin local ring and $B_{0}$ is of homological dimension $\leqslant 2$ with $A$ noetherian (the noetherian hypothesis can be dropped for an infinitesimal deformation). The other case is the case of an infinitesimal deformation where the defining ideal for $B_{0}$ is a local complete intersection. The first case is done in Section 2 while the second is in Section 3.

\section{Some Remarks on Flatness}

We begin with a well-known observation.

Received 19 July 1988

Copyright Clearance Centre, Inc. Serial-fee code: $0004-9729 / 89 \$ \$ 2.00+0.00$. 
LEMma 1.1. Let $A$ be a ring and

$$
0 \rightarrow K \rightarrow L \rightarrow M \rightarrow 0
$$

an exact sequence of $A$-modules with $L$ flat. Then:

(1) given an ideal $\mathcal{A} \subseteq A, \operatorname{Tor}_{1}^{A}(M, A / \mathcal{A})=0$ if and only if $\mathcal{A} L \cap K=\mathcal{A} K$;

(2) $M$ is flat if and only if $\mathcal{A} L \cap K=\mathcal{A} K$ for every ideal $\mathcal{A}$ of $A$.

Proof: Tensor the sequence with $A / \mathcal{A}$ and note that $\operatorname{Tor}_{1}^{A}(L, A / \mathcal{A}) \cong 0$ so $\operatorname{Tor}_{1}^{A}(M, A / \mathcal{A})=(\mathcal{A} L \cap K) / \mathcal{A} K$. (1) follows, and (2) is a consequence of (1).

Definition: Given a ring $A$, an ideal $\mathcal{A}$ of $A$, and an $A$-module $M$, we say that $M$ is idcalwise separated for $\mathcal{A}$ if for every finitely generated ideal $I$ of $A$, $\cap_{n=1}^{\infty} \mathcal{A}^{n}\left(I \otimes_{A} M\right)=0$.

An important special case in which $M$ is ideal-wise separated for $\mathcal{A}$ is when there is a noetherian $A$-algebra $B$ so that $M$ is a finite $B$-module and $A B$ is contained in the Jacobson radial of $B$.

TheOREM 1.2. Assume that there is an exact sequence of $A$-modules

$$
0 \rightarrow K \rightarrow L \rightarrow M \rightarrow 0
$$

where $L$ is flat. Let $\mathcal{A}$ be an ideal in $A$, and suppose that either:

(i) $\mathcal{A}$ is nilpotent or

(ii) $A$ is noetherian and $M$ is idealwise separated for $\mathcal{A}$.

Then the following are equivalent:

(1) $M$ is A-flat;

(2) $M / \mathcal{A} M$ is $(A / \mathcal{A})$-flat and $\mathcal{A} L \cap K=\mathcal{A} K$.

Proof: Follows from $[6,(20 . \mathrm{C})]$ and Lemma 1.1.

In general it is not true that condition (2) implies that $M$ is $A$-flat, even when $S$ is a local ring.

Example. Let $k$ be a field. $k[u, v, w]$ the polynomial ring in 3 variables. Let $\mathcal{M}=(u, v, w)$ and set $A=k[u, v, w]_{\mathcal{M}}$, the localization at $\mathcal{M}$. Take $M=A[x, y] /$ $(u x-1, v y-w)$. Then we have an exact sequence as in Theorem 1.2 with $L=A[x, y]$ and $K=(u x-1, u y-w)$. Note that $M / \mathcal{M} M=(0)$ and $\mathcal{M} L \cap K=\mathcal{M} K$ since $\mathcal{M}$ and $K$ are comaximal. However, $(v, w) L \cap K \neq(v, w) K$ so $M$ is not flat by Lemma $1(2)$.

Let $A$ be a ring, $\mathcal{A}$ an ideal of $A, A_{0}=A / \mathcal{A}$, and $B_{0}$ a finitely generated $A_{0}$-algebra which is flat over $A_{0}$. In order to deform $B_{0} / A_{0}$ over $A$ we must find a 
flat $A$-algebra, $B$, such that $B / \mathcal{A} B=B_{0}$. If $B_{0}=A_{0}\left[x_{1}, \ldots, x_{m}\right] / I_{0}$ one can easily find an ideal $I$ such that if $B=A\left[x_{1}, \ldots, x_{m}\right] / I$ then $B / \mathcal{A} B=B_{0}$. Simply choose preimages for the generators for $I_{0}$ under the map $A\left[x_{1}, \ldots, x_{m}\right] \rightarrow A_{0}\left[x_{1}, \ldots, x_{m}\right]$. The difficulty is choosing $I$ so that $B$ is $A$-flat. Later on we will demonstrate that under certain circumstances $I$ can be chosen so that $\mathcal{A} A\left[x_{1}, \ldots, x_{m}\right] \cap I=\mathcal{A} I$. Hence we are in the context of Theorem 1.2 with $M=B, L=A\left[x_{1}, \ldots, x_{m}\right], K=I$, lacking only hypothesis (i) or (ii). As the example demonstrates, we camnot in general conclude flatness. However, we do have:

Theorem 1.3. Let $A$ be a noetherian ring, $B=A\left[x_{1}, \ldots, x_{m}\right] / I, \mathcal{A}$ an ideal of $A, A_{v}=A / \mathcal{A}$, and $B_{0}=B / \mathcal{A} B$. Suppose that $B_{0}$ is $A_{0}$-flat and $\mathcal{A} A\left[x_{1}, \ldots, x_{m}\right] \cap$ $I=\mathcal{A} I$. Then there is an $f \in B$ which is a unit modulo $\mathcal{A}$ and such that $B_{f}$ is $A$-flat.

Hence, there is a finitely generated $A$-algebra $B^{\prime}=B_{f}$ which is a deformation of $B / \mathcal{A} B$ over $A$.

Proof: Let $P$ be a prime in $B, Q \subset A\left[x_{1}, \ldots, x_{m}\right]$ the inverse image of $P$ under the surjection $A\left[x_{1}, \ldots, x_{m}\right] \rightarrow B$, and let $C=A\left[x_{1}, \ldots, x_{m}\right]_{Q}$. Then $\mathcal{A} C \cap I C=$ $A I C$ by the hypothesis.

If $\mathcal{A} B \subseteq P$ then $B_{P}$ is flat by Theorem 1.2. By [ 6 , Theorem 5.3] there is an open set $U$ in spec $(B)$ containing $V(\mathcal{A B})$ such that $B_{P}$ is flat for all $P \in U$. We wish to find an affine open set with the same property. Let $\operatorname{spec}(B)-U=V(J)$. Since $V(J) \cap V(\mathcal{A B})=0$, we have $J+\mathcal{A} B=B$. Choose $f \in J$ so that $(f)+\mathcal{A} B=B$. Then $B_{f}$ is $A$-flat by $[6,(3 \mathrm{j})]$.

\section{Deformations in Homological Dimension $\leqslant 2$}

Our object is to prove:

Theorem 2.1. Given an Artin local ring $A_{0}$ and a finitely generated $A_{0}$-algebra $A_{0}\left[x_{1}, \ldots, x_{m}\right] / I_{0}$, suppose that $B_{0}$ is $A_{0}$-flat and that $d h_{A_{0}\left[x_{1}, \ldots, x_{m}\right]}\left(B_{0}\right) \leqslant 2$. Let $A$ be a ring and $\mathcal{M} \subseteq A$ an ideal such that $A / \mathcal{M} \cong A_{0}$. Suppose that either:

(i) $\mathcal{M}$ is nilpotent or

(ii) $A$ is noetherian.

Then there is a deformation $B$ of $B_{0} / A_{0}$ over $A$.

Proof: Let $R_{0}=A_{0}\left[x_{1}, \ldots, x_{m}\right]$. By hypothesis there is a resolution of the form:

$$
0 \rightarrow P_{2} \rightarrow P_{1} \rightarrow R_{0} \rightarrow B_{0} \rightarrow 0
$$

where $P_{1}$ and $P_{2}$ are projective $R_{0}$-modules of finite rank. But $P_{1}$ and $P_{2}$ are free modulo the maximal ideal $N$ of $A_{0}$ by [7] or [8]. Since the maximal ideal is nilpotent 
$P_{1}$ and $P_{2}$ must be free. (Apply Nakayama's Lemma to the preimage of a basis mod $N$ ). Therefore we have a resolution

$$
0 \rightarrow R_{0}^{r-1} \stackrel{\phi_{2}}{\rightarrow} R_{0}^{r} \stackrel{\phi_{1}}{\rightarrow} R_{0} \rightarrow B_{0} \rightarrow 0
$$

We now apply a standard technique using Burch's Theorem. We can find no reference that precisely fits our situation so we give the details (see [1] and [8]). Let $M=\left[a_{i j}\right]$ be the $r \times(r-1)$ matrix that represents $\phi_{2}$ and $\left[f_{1}, \ldots, f_{r}\right]$ the matrix representation for $\phi_{1}$. Let $f_{i}$ be the minor of $M$ obtained by omitting the $i$ th row of $M$. By Burch's Theorem ([4, p.148, problem 8]) there is a regular element $f \in R_{0}$ such that $f d_{i}=f_{i}$. We let $R=A\left[x_{1}, \ldots, x_{m}\right]$ and define $\psi_{2}: R^{r-1} \rightarrow R^{r}$ by lifting $M$ to a matrix $\bar{M}=\left[\tilde{a}_{i j}\right]$ over $R$. Let $\tilde{f}$ be a lifting for $f$ and define $g_{i}=\tilde{f} \tilde{d}_{i}$ where $\tilde{d}_{i}$ is the minor of $\tilde{M}$ obtained by omitting the $i$ th row. Then $\psi_{1} \circ \psi_{2}=0$ since, for any $k$

$$
g_{1} \tilde{a}_{1 k}+\ldots+g_{r} a_{r k}=\tilde{f} \operatorname{deg}\left[\begin{array}{cccccc}
a_{1 k} & a_{11} & \ldots & a_{1 k} & \ldots & a_{1 k-1} \\
\vdots & \vdots & \ldots & \vdots & \ldots & \vdots \\
a_{r k} & a_{r 1} & \ldots & a_{r k} & \ldots & a_{r k-1}
\end{array}\right]=0
$$

We now use the following lemma:

LEMMA 2.2. Let $R$ be a ring, $\mathcal{A} \subseteq R$ an ideal, and $R_{0}=R / \mathcal{A}$. Suppose we have a diagram:

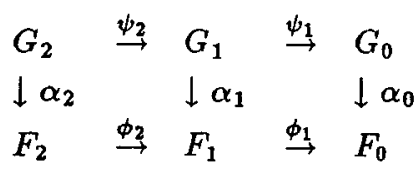

with the bottom row an exact sequence of $R_{0}$-modules and the top row a complex of $R$-modules (that is $\psi_{1} \circ \psi_{2}=0$ ). Suppose further that, for each $i, F_{i}=F_{i} \otimes_{R} R_{0}$ with $\alpha_{i}$ the canonical projection.

Then $\mathcal{A} G_{0} \cap \operatorname{im}\left(\psi_{1}\right)=\mathcal{A i m}\left(\psi_{1}\right)$.

Proof: Choose $z \in \mathcal{A} G_{0} \cap \operatorname{im}\left(\psi_{1}\right)$. Then $z=\psi_{1}(w)$ for some $w \in G_{1}$. But $z \in \mathcal{A} G_{0} \Rightarrow \alpha_{0}(z)=0$. Hence $\phi_{1} \alpha_{1}(w)=\alpha_{0} \psi_{1}(w)=0$. By exactness $\alpha_{1}(w)=\phi_{1}\left(u^{\prime}\right)$ for some $u^{\prime} \in F_{2}$. Pick $u \in G_{2}$ with $\alpha_{2}(u)=u^{\prime}$. Then $\alpha_{1}(w)=\alpha_{1}\left(\psi_{2}(u)\right)$ and $w-\psi_{2}(u) \in \operatorname{ker} \alpha_{1}=\mathcal{A} G_{1}$ and $\psi_{1}\left(w-\psi_{2}(u)\right) \in \mathcal{A} \operatorname{im}\left(\psi_{1}\right)$. But $\psi_{1}\left(w-\psi_{2}(u)\right)=$ $\psi_{1}(w)=z$ and $z \in \mathcal{A i m}\left(\psi_{1}\right)$.

We have shown that

$$
\mathcal{A} G_{0} \cap\left(\psi_{1}\right) \subseteq \mathcal{A} \operatorname{im}\left(\psi_{1}\right)
$$

The reverse inclusion is obvious. 
Now applying the lemma to the sequence

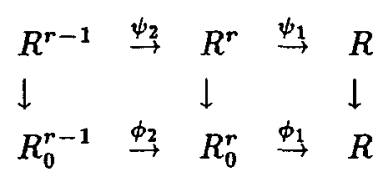

and letting $1=\operatorname{im}\left(\psi_{1}\right)$ we get that $\mathcal{M} R \cap 1=\mathcal{M} 1$. Hence, by Theorem 1.3 in the noetherian case or Theorem 1.2 in the nilpotent case we get the result.

Corollary 2.3. Let $A_{0}$ be an Artin local ring and $B_{0}=A_{0}\left[x_{1}, x_{2}\right] / I_{0}$ be a flat $A_{0}$-algebra. Let $A$ be a ring with an ideal $\mathcal{M}$ such that either:

(i) $\mathcal{M}$ is nilpotent, or

(ii) $A$ is noetherian.

Then there is a deformation $B$ of $B_{0} / A_{0}$ over $A$.

(Note: There are examples of rings $A, A_{0}$ as above and $B_{0}=A\left[x_{1}, x_{2}, x_{3}, x_{4}, x_{5}, x_{6}\right] / I_{0}$ for which there is no deformation of $B_{0} / A_{0}$ over A. (See [2] or [3].))

Proof: We need show only that $d h_{R_{0}}\left(B_{0}\right) \leqslant 2$ with $R_{0}=A_{0}\left[x_{1}, x_{2}\right]$. Consider the exact sequence

$$
0 \rightarrow K \rightarrow R_{0}^{n} \rightarrow R_{0} \rightarrow B_{0} \rightarrow 0 .
$$

Let $k$ be the residue field of $A_{0}$.

By the flatness of $B_{0}$ we have

$$
0 \rightarrow K \otimes A_{0} k \rightarrow R_{0}^{n} \otimes_{A_{0}} k \rightarrow R_{0} \otimes_{A_{0}} k \rightarrow B_{0} \otimes_{A_{0}} k \rightarrow 0
$$

$R_{0} \otimes_{A_{0}} k=k\left[x_{1}, x_{2}\right]$ which has global dimension 2 so $K \otimes_{A_{0}} k$ is projective (hence free) and that implies that $K$ is free.

3. INFINITESIMAL DEFORMATIONS OF LOCAL COMPLETE INTERSECTIONS

DEFINITION: Let $R$ be a ring and $I \subset R$ an ideal. $I$ is a local complete intersection if, for every $P \in \operatorname{spec}(R)$ with $I \subseteq P, I R_{P}$ is generated by an $R_{P}$-regular sequence.

Our objective is to prove the following theorem:

Theorem 3.1. Given a noetherian ring $A_{0}$ and a finitely generated $A_{0}$-algebra $B_{0}=A_{0}\left[x_{1}, \ldots, x_{n}\right] / I_{0}$, suppose that $B_{0}$ is $A_{0}$-flat and that $I_{0}$ is a local complete intersection in $A_{0}\left[x_{1}, \ldots, x_{n}\right]$. Let $A$ be a ring and $\mathcal{A} \subseteq A$ an ideal in $A$ such that $A / \mathcal{A}=A_{0}$ and $\mathcal{A}$ is nilpotent. Then there is a finitely generated A-algebra $B$ such that $B$ is a deformation of $B_{0} / A_{0}$ over $A$. 
Furthermore, if $B$ is any deformation of $B_{0} / A_{0}$ over $A$ and $B=A\left[y_{1}, \ldots, y_{n \mathbf{r}}\right] / I$. Then $I$ is a local complete intersection in $A\left[y_{1}, \ldots, y_{m}\right]$.

In order to prove Theorem 3.1 we need to use the upper cotangent functor $T^{i}(B / A, M)$ associated with a ring homomorphism $A \rightarrow B$ and a $B$-module $M$. (see [5]). We state two results from [5].

Theorem 3.2. ([5, 3.2.2]) Let $A$ be a noetherian ring and $B$ a finitely generated $A$-algebra. The following conditions are equivalent:

(a) $B=A\left[x_{1}, \ldots, x_{n}\right] / I$ where $I$ is a local complete intersection in $A\left[x_{1}, \ldots, x_{n}\right]$;

(b) for any surjection $A\left[y_{1}, \ldots, y_{m}\right] \rightarrow B$ the kernel is a local complete intersection of $A\left[y_{1}, \ldots, y_{m}\right]$;

(c) $T^{2}(B / A, M)=0$ for every $B$-module $M$.

Before stating the next theorem we need a little explanation. Consider a sequence of ring homomorphisms $A \rightarrow A_{0} \rightarrow B_{0}$ where $A_{0}=A / \mathcal{A}$ with $\mathcal{A}^{2}=0$. This gives a long exact change of rings sequence of cotangent functors. In particular, we have a map

$$
\delta: T^{1}\left(A_{0} / A, \mathcal{A} \otimes_{A_{0}} B_{0}\right) \rightarrow T^{2}\left(B_{0} / A_{0}, \mathcal{A} \otimes_{A_{0}} B_{0}\right)
$$

(Note that $\mathcal{A}=\mathcal{A} / \mathcal{A}^{2}$ is an $A_{0}$-module). It is known that $T^{1}\left(A_{0} / A, \mathcal{A} \otimes_{A_{0}} B_{0}\right) \cong$ $\operatorname{End}_{B_{0}}\left(\mathcal{A}_{0}, \mathcal{A} \otimes_{A_{0}} B\right)$. Hence there is a canonical element $\delta(1) \in T^{2}\left(B_{0} / A_{0}, \mathcal{A} \otimes_{A_{0}} B_{0}\right)$.

Theorem 3.3. ([5, 4.3.3]) Let $A$ be a ring and let $A_{0}-A / \mathcal{A}$ with $\mathcal{A}^{2}=0$. Suppose that $B_{0}$ is a flat $A_{0}$-algebra. Then there is a deformation of $B_{0} / A_{0}$ over $A$ if and only if $\delta(1)=0$ in $T^{2}\left(B_{0} / A_{0}, \mathcal{A} \otimes A_{0} B_{0}\right)$.

Proof of TheOREM 3.1: We first prove the last statement. So assume that $B$ is a deformation of $B_{0} / A_{0}$ over $A$. By Theorem 3.2 it is sufficient to prove that $T^{2}(B / A, M)=0$ for every $B$-module $M$. Since $\mathcal{A}$ is nilpotent there is an integer $k$ such that $\mathcal{A}^{k} M=0$. Our proof is by induction on $k$.

For $k=1, M$ is a $B / A B \cong B_{0}$-module. Since $B$ is flat over $A$ we can conclude by $[5,2.3 .2]$ that $T^{2}(B / A, M) \cong T^{2}\left(B_{0} / A_{0}, M\right)$. Therefore, by Theorem $3.2, I$ is a local complete intersection.

Now suppose $k>1$ and consider the exact sequence of $B$-modules

$$
0 \rightarrow \mathcal{A} M \rightarrow M \rightarrow M / \mathcal{A} M \rightarrow 0
$$

By the induction hypothesis $T^{2}(B / A, \mathcal{A} M)=T^{2}(B / A, M / \mathcal{A} M)=0$ and by the long exact sequence of $T^{i}([5,2.3 .6]) T^{2}(B / A, M)=0$ and $I$ is a local complete intersection. 
Now assume that $B_{0}, A_{0}$, and $A$ are as in the theorem and we wish to show that a deformation exists. Let $k$ be such that $\mathcal{A}^{k}=0$. Again, we proceed by induction on $k$. If $k \leqslant 2$ we are done by Theorem 3.2 and Theorem 3.3 so assume that $k>2$ and let $B_{k-1}$ be a deformation of $B_{0} / A_{0}$ over $A_{k-1}=A / \mathcal{A}^{k-1}$ of the form $B_{k-1}=A_{k-1}\left[y_{1}, \ldots, y_{n}\right] / I_{k-1}$. By the first part of the proof $I_{k-1}$ is a local complete intersection and we can find a finitely generated deformation of $B_{k-1} / A_{k-1}$ over $A$.

\section{REFERENCES}

[1] D. Buchsbaum and D. Eisenbud, Lifting modules and a theorem on finite free resolutions, in Ring Rheory (Academic Press, New York, 1972).

[2] M. Hochster, 'An obstruction to lifting cyclic modules', Pacific J. Math 61 (1975), 457-463.

[3] J.W. Hoffman, 'Counterexamples to the lifting problem for singularities', Comm. Algebra 11 (1983), 523-549.

[4] I. Kaplasky, Commutative Rings (Univ, of Chicago Press, 1974).

[6] S. Lichtenbaum and M. Schlessinger, 'The cotangent complex of a morphism', Trans. Amer. Math. Soc 128 (1967), 41-70.

[6] H. Matsumura, Commutative Algebra (2nd ed.) (Benjamin Cumming, Reading Mass, 1980).

[7] D. Quillen, 'Projective modules over polynomial rings', Invent. Math. 36 (1976), $167-171$.

[8] M. Schaps, 'Deformations of Cohen-Macauley schemes of codimension 2 and non-singular deformations of space curves', Amer. J. Math 89 (1977), 669-685.

[ө] A.A. Suslin, 'Projective modules over a polynomial ring are free', Dokl. Akad. Nauk. S.S.S.R. 229 (1976), 1063-1066. Soviet Math. Dokl 17 (1976), 1160-1164.

Department of Mathematical Sciences

Virginia Commonwealth University

Richmond, VA 23284

United States of America 